\title{
Acute Response of Sclerostin to Whole-body Vibration with Blood Flow Restriction
}

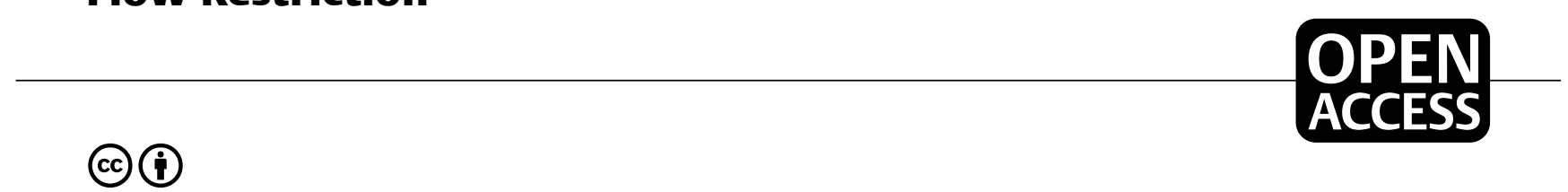

Authors

Kyle S Gapper, Sally Stevens, Rona Antoni, Julie Hunt, Sarah J Allison

\author{
Affiliations \\ Department of Bioscience \& Medicine, University of Surrey, \\ Guildford, United Kingdom of Great Britain and Northern \\ Ireland
}

\section{Key words}

bone metabolism, bone turnover markers, blood flow restriction, vibration, acute response

accepted 27.02.2021

published online 11.05 .2021

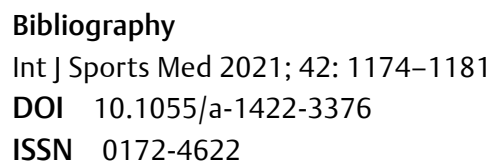

\begin{abstract}
Blood flow restriction may augment the skeletal response to whole-body vibration. This study used a randomised, crossover design to investigate the acute response of serum sclerostin and bone turnover biomarkers to whole-body vibration with blood flow restriction. Ten healthy males (mean \pm standard deviation; age: $27 \pm 8$ years) completed two experimental conditions separated by 7 days: (i) whole-body vibration (10 1-minute bouts of whole-body vibration with $30 \mathrm{~s}$ recovery) or (ii) whole-body vibration with lower-body blood flow restriction (10 cycles of $110 \mathrm{mmHg}$ inflation with $30 \mathrm{~s}$ deflation during recovery). Fasting blood samples were obtained immediately before and immediately after exercise, then 1 hour, and 24 hours after exercise. Serum samples were analysed for sclerostin, cross-linked C-terminal telopeptide of type I collagen, and bone-specific alkaline phosphatase. There was a significant time $\times$ condition interaction for bone-specific alkaline phosphatase ( $p=0.003)$; bone-specific alkaline phosphatase values at 24 hours post-exercise were significantly higher following whole-body vibration compared to combined whole-body vibration and blood flow restriction $(p=0.028)$. No significant time $\times$ condition interaction occurred for any other outcome measure $(p>0.05)$. These findings suggest that a single session of whole-body vibration combined with blood flow restriction does not significantly affect serum sclerostin or bone turnover biomarkers.
\end{abstract}

\section{Introduction}

Blood flow restriction (BFR) involves the application of a tourniquet system to the proximal portion of a limb to partially restrict arterial inflow and fully restrict venous outflow [1]. Mechanical compression of the underlying vasculature induces localised hypoxia distal to the placement of the cuff while causing blood pooling within the capillaries of the occluded limbs via diminution of venous blood flow [2]. This technique has typically been applied to resistance exercise to augment the muscle hypertrophic and strength responses to low-load resistance training $[1,3,4]$. However, there is emer- ging evidence to suggest that BFR exercise may also provide a novel stimulus to bone.

Bone turnover markers are proteins or degradation products released during the process of bone remodelling and include markers of bone formation and bone resorption [5]. Bone turnover markers have been associated with bone mineral density (BMD) [6, 7] and fracture risk [8] and have been used to evaluate exercise and training effects [9]. Some studies have investigated the response of bone turnover markers following BFR exercise [10-12]. BFR increased the expression of bone formation biomarkers such as bonespecific alkaline phosphatase (B-ALP) and/or decreased bone re- 
sorption biomarkers such as the cross-linking telopeptides of type I collagen (CTX) when combined with walking or resistance exercise [10-12]. Interestingly, one study reported a reduction in CTX 30 minutes following low-load BFR resistance exercise that was of a greater magnitude than the reductions reported following unrestricted low-load resistance exercise [11]. Although these prior findings are encouraging, the application of BFR to date has been limited to walking or resistance exercise and the acute osteogenic response of BFR exercise has only been assessed by markers of bone formation and resorption.

Osteocytes are the primary bone cells that sense mechanical strain via fluid flow shear stress through the lacuna-canalicular network and changes in interstitial hydrostatic pressure [13-15]. One potentially important mechanism by which mechanical stimuli influence osteocyte activity is through regulating sclerostin, an osteocyte-derived secreted glycoprotein, which inhibits bone formation through inhibition of the Wnt/ $\beta$-catenin pathway $[16,17]$. There have been few human studies to date reporting the response of sclerostin to acute exercise, although current evidence suggests that sclerostin levels increase following an acute bout of physical activity [18, 19], high-intensity interval exercise [20], jumping [21], whole-body vibration (WBV) [22], and combined high-intensity resistance training and WBV [23].

BFR applied to WBV could provide a new mode of stimulation to optimise bone health in populations that may have difficulty performing high-impact or high-intensity resistance exercise, such as untrained individuals, older adults, or those undergoing musculoskeletal rehabilitation. WBV provides mechanical stimuli to bone in the form of vertical oscillation, which is adequate to increase fluid flow in bone and produce an osteogenic signal [24]. Since elevations in intramedullary pressure and changes in bone fluid flow is one of the mechanisms through which BFR can stimulate bone adaptation [25], adding a BFR stimulus to a traditional WBV protocol may potentially exert an additive osteogenic effect. However, the response of sclerostin and bone turnover makers to combined WBV and BFR is yet to be explored. Such investigations could provide insight into the specificity of the bone response to BFR exercise in the short term. The primary aim of this study was to investigate the response of serum sclerostin and bone turnover biomarkers to an acute bout of WBV superimposed with BFR. It was hypothesised that combined WBV and BFR would transiently increase serum sclerostin concentrations to a greater extent than that resulting from WBV alone.

\section{Materials and Methods}

\section{Participants}

Ten healthy untrained males aged $18-39$ years volunteered to participate in this investigation (see $>$ Table 1 for participant demographics). Participants were excluded from the study if they met any of the following exclusion criteria: (i) current smoker; (ii) a $\mathrm{BMI} \geq 30 \mathrm{~kg} / \mathrm{m}^{2}$; (iii) a history of cardiovascular (including hypertension), metabolic, haematological, neurological, or musculoskeletal disease or injury; ( $v$ ) consumed medication or supplements known to influence bone status; and (vi) performed weight-bearing endurance or resistance training more than three times per week over the preceding 12 months. Before initiating the study, all participants provided written informed consent and completed a health history questionnaire to document information about exercise training status and to detect potential risk factors that may be aggravated by performing the type of activity in the study. Participants also completed the bone-specific physical activity questionnaire (BPAQ) to quantify the degree of activity-related skeletal loading from current (previous 12 months) and past (from one year of age) activities [26]. This investigation was granted a favourable ethical opinion from the University of Surrey Ethics Committee and conformed to the ethical requirements stipulated by the International Journal of Sports Medicine [27].

\section{Experimental design}

A randomised, crossover design was used to investigate the acute response of serum sclerostin and bone turnover biomarkers to a single session of WBV exercise with and without BFR. Participants attended the laboratory on five separate occasions (familiarisation, two experimental trials, and two 24-h follow-up visits), with each experimental trial separated by a minimum of 7 days. A schematic overview of the study design is presented in $\mathbf{~ F i g . ~} \mathbf{1}$.

During the first visit to the laboratory, participants completed all paperwork, provided measures of height (Seca 220; Seca, Hamburg, Germany), body mass (Seca 761; Seca), and blood pressure (Omron M2; OMRON Healthcare, Kyoto, Japan), and were familiarised with the exercise equipment and protocol. Participants then attended the laboratory on four more occasions to complete two experimental conditions in a random counterbalanced order: (i) whole-body vibration only (WBV), and (ii) whole-body vibration with blood flow restriction (WBV+BFR). Each experimental trial had a follow-up visit 24 hours later. Participants attended and completed all experimental trials and follow-up visits having undertaken an overnight fast of a minimum of 8 hours. Each exercise bout was performed at the same time of day. Participants recorded their dietary and activity habits during the 72 hours preceding the first experimental trial and were asked to replicate these habits before the second trial. Total energy, macronutrient, calcium, and vitamin D intake were quantified using nutrition analysis software (Nutritics LTD, Dublin, Ireland). Participants abstained from strenuous physical activity, caffeine, and alcohol consumption for at least 72 hours before each visit.

- Table 1 Participant demographics $(n=10)$.

\begin{tabular}{|c|c|}
\hline & Mean \pm SD \\
\hline Age (years) & $27 \pm 8$ \\
\hline Body mass $(\mathrm{kg})$ & $78.5 \pm 9.4$ \\
\hline Height (cm) & $179.2 \pm 5.2$ \\
\hline BMI $\left(\mathrm{kg} / \mathrm{m}^{2}\right)$ & $24.5 \pm 2.8$ \\
\hline \multicolumn{2}{|c|}{ Resting blood pressure $(\mathrm{mmHg})$} \\
\hline Systolic blood pressure & $129 \pm 8$ \\
\hline Diastolic blood pressure & $71 \pm 8$ \\
\hline \multicolumn{2}{|l|}{ BPAQ scores } \\
\hline Current BPAQ score & $6.1 \pm 5.9$ \\
\hline Past BPAQ score & $39.2 \pm 30$ \\
\hline
\end{tabular}




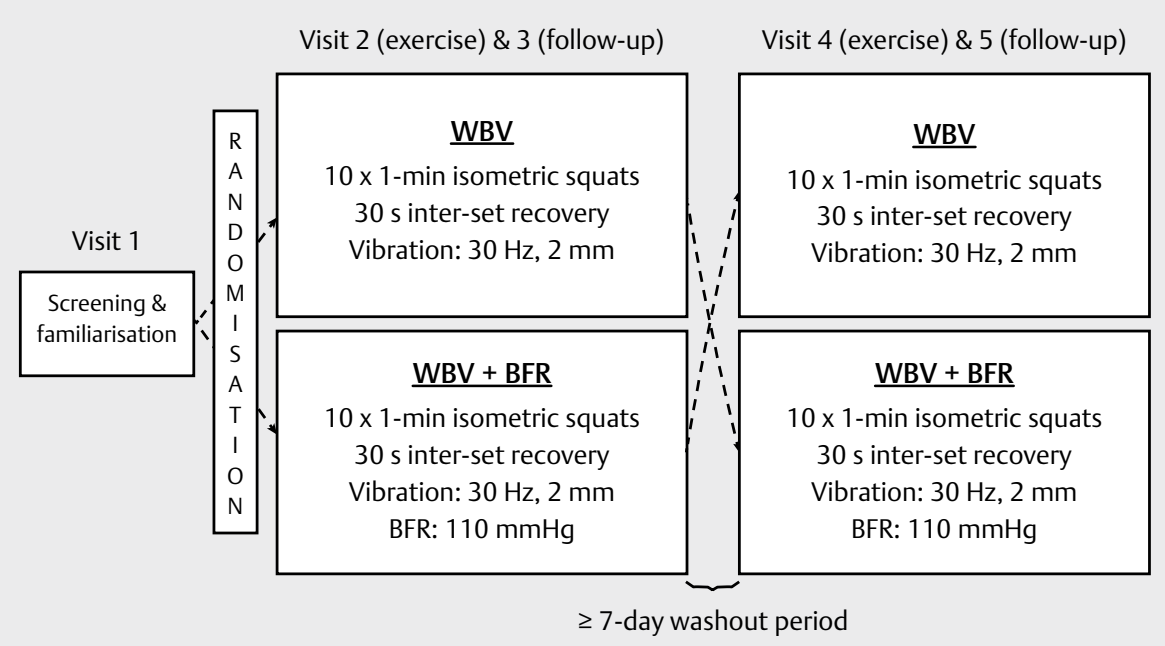

- Fig. 1 Schematic overview of the study design. WBV, whole-body vibration; WBV + BFR, whole-body vibration with blood flow restriction.

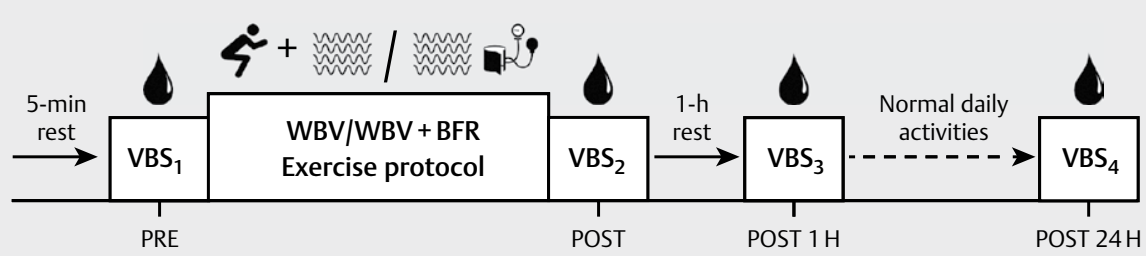

- Fig. 2 Schematic overview of the experimental procedures for the study. WBV, whole-body vibration; WBV+BFR, whole-body vibration; $V_{B S}{ }_{1-4}$, venous blood sample 1-4; PRE, pre-exercise; POST, immediately post-exercise; POST 1H, 1-hour post-exercise; POST 24H, 24 hours post-exercise.

\section{Loading protocols}

A schematic overview of the experimental protocol is presented in

Fig. 2.

\section{Whole-body vibration (WBV)}

Participants underwent ten 1-minute bouts of vibration exercise with 30 s recovery between repetitions; the total duration of vibration was 10 minutes. Participants stood barefoot on a synchronous vibration platform (Power Plate NEXT generation; Performance Health Systems UK Ltd., London UK) in an isometric semi-squat position with a relative knee joint angle of $130^{\circ}$. The knee joint angle was measured manually using a goniometer to maintain consistency between trials. Participants performed each squat with their hands on their hips and were instructed to keep balanced foot plantar pressure between the rearand forefoot. A semi-squat position with static posture was chosen as it has been shown to improve bone mineral density when performed with WBV while minimising transmission to the head [28]. The vertical vibration stimulus was administered at a frequency of $30 \mathrm{~Hz}$ with a peak-to-peak displacement (i. e. displacement from the lowest to the highest point of the total vibration excursion) of $2 \mathrm{~mm}$ as reported by the manufacturer. A "low" setting was used on the Power Plate to achieve the desired vibration amplitude. Expressed as a multiple of Earth's gravity, the peak acceleration of the vibration stimulus was $3.6 \mathrm{~g}$ based the equation by Rauch et al. [29]. This vibration intensity (e. g. frequency and peak-to-peak amplitude) is similar to that used in other acute studies that have documented positive physiological changes during and following combined WBV and BFR [30,31].

Whole-body vibration with blood flow restriction (WBV + BFR)

The WBV + BFR condition used the WBV protocol described above with the addition of lower-limb BFR. A 13-cm-wide pneumatic cuff (Model SC12LTM; Hokanson, Bellevue, WA, USA) was applied to the most proximal portion of each thigh (distal to the inguinal fold) and inflated (E20 Rapid Cuff Inflator and AG101 Cuff Inflator Air Source; Hokanson) to $110 \mathrm{mmHg}$ for the entire 1-minute duration of each repetition. Both thigh cuffs were fully deflated during each 30 -second recovery interval. The selected inflation pressure was similar to earlier work [32] showing that the absolute pressure required to occlude lower-body arterial blood flow is approximately $40 \%$ lower when using wider cuffs compared to narrow cuffs in a sample of healthy, young adults. At $110 \mathrm{mmHg}$, we estimate that the degree of blood flow restriction attained was approximately $60 \%$ [33]. An intermittent BFR protocol (i. e. cuff pressure released between exercises/exercise sets) was selected based on longer-term studies documenting its ability to influence bone changes [34, 35]. Our decision to use short inflation periods draws support from prior evidence that bone responds to mechanical stimulus that is brief and 
interspersed with short rest periods [36]. It is also important to note that in pilot testing, participants were unable to complete the desired exercise volume with the cuff inflated throughout, so it was not practical to use a continuous BFR protocol at this pressure.

\section{Blood sampling}

Venepuncture blood samples from both experimental trials were collected between 07:00 and 09:00 hours and the time of blood draw was consistent for each participant. Samples were obtained by a qualified phlebotomist before (PRE), immediately after (POST), 1-hour after (POST 1H), and 24 hours after (POST 24H) the exercise session. Each blood sample was drawn from an antecubital vein and gathered into 4-ml serum separator tubes. Collected samples were allowed to clot at room temperature for 30 minutes. Serum was separated by centrifugation at $2500 \mathrm{rpm}$ for 10 minutes, and serum aliquot was frozen at $-20^{\circ}$ until the assay analyses were performed. All samples were analysed in batch upon trial completion.

\section{Bone biomarker analysis}

Serum sclerostin was determined using the TECO Medical high-sensitivity enzyme immunoassay (EIA) (Quidel Corporation, San Diego, CA, USA). Serum CTX and B-ALP were assessed using an enzymelinked immunosorbent assay (ELISA) (CTX: Immunodiagnostic Systems, Tyne \& Wear, UK; B-ALP: MicroVue BAP EIA; Quidel Corporation). The intra- and inter-assay coefficient of variation for sclerostin, CTX and B-ALP ranged between 3.7-4.2\% and 4.3-4.8\%, 1.7$3.0 \%$ and $2.5-10.9 \%, 3.9-5.8 \%$ and $5.0-7.6 \%$ respectively, as stated by the manufacturer. All samples were run in duplicate and had undergone a single freeze/thaw cycle.

\section{Statistical analysis}

Statistical analyses were performed using IBM SPSS Statistics 25.0 for Windows (IBM Corp., Armonk, NY, USA). Normality of the data set was evaluated using the Shapiro-Wilk test. Between-trial differences in overall energy intake and protein, carbohydrate, calcium, and vitamin $D$ consumption were assessed using paired-samples ttests. A two-way (condition $\times$ time) repeated-measures analysis of variance (ANOVA) was used to determine the effects of the experimental conditions over time on serum sclerostin, CTX, and B-ALP concentrations. Mauchly's test of sphericity was conducted to assess for sphericity of data, and Greenhouse-Geisser adjustments were made to correct for sphericity violations. Any significant main effects of time were assessed using separate one-way repeated measures ANOVAs. When significant condition $\times$ time interactions occurred, paired-samples t-tests with a Bonferroni correction for multiple comparisons were conducted at each level of time to locate the time point at which significant effects were present.

Effect sizes were computed as Cohen's d or partial eta-squared $\left(\eta_{p}{ }^{2}\right)$. Cohen's $d$ was calculated as the mean difference of the two values at a given time point divided by the pooled SD of the two values [37]. Partial eta-squared was calculated as the sum of squares of the effect divided by the sum of squares of the same effect and its associated error variance [38]. All data are presented as mean $\pm 95 \%$ confidence intervals unless otherwise stated. Statistical significance was accepted at $\mathrm{p}<0.05$.

\section{Results}

\section{Participant demographics}

Participant demographics are summarised in $>$ Table 1.

\section{Dietary intake}

Overall energy intake $(p=0.48)$ and protein $(p=0.78)$, carbohydrate $(p=0.53)$, calcium $(p=0.32)$, and vitamin $D(p=0.14)$ consumption were similar over the 72 hours preceding the two experimental trials. Descriptive food diary data are presented in > Table 2.

\section{Bone biomarker responses}

An overview of all bone biomarker response data is provided in - Table 3.

\section{Sclerostin}

There was no statistically significant main effect of condition $\left(p=0.14 ; \eta_{p}^{2}=0.22\right)$ or time $\left(p=0.43 ; \eta_{p}^{2}=0.08\right)$, and no significant condition $\times$ time interaction $\left(p=0.68 ; \eta_{p}{ }^{2}=0.05\right)$ for serum sclerostin. The absolute mean change of serum sclerostin from preexercise values is presented in $\mathbf{F i g}$. $\mathbf{3 a}$.

\section{CTX}

A significant main effect of time $\left(p=0.018 ; \eta_{p}^{2}=0.31\right)$ but not condition $\left(p=0.17 ; \eta_{p}{ }^{2}=0.20\right)$ was revealed for serum CTX. No condition $\times$ time interaction occurred $\left(p=0.27 ; \eta_{p}{ }^{2}=0.13\right)$. Despite indications of a significant main effect of time, post-hoc one-way ANOVAs revealed no significant difference between time points for either condition $(p>0.05)$. The absolute mean change of serum CTX from pre-exercise values is presented in $\mathbf{F i g . ~} \mathbf{3 b}$.

\section{B-ALP}

There was no significant main effect of condition $(p=0.94$; $\left.\eta_{p}{ }^{2}=0.001\right)$ or time $\left(p=0.086 ; \eta_{p}^{2}=0.24\right)$ for serum B-ALP; however, there was a significant condition $\times$ time interaction $(p<0.001$; $\eta_{p}{ }^{2}=0.49$ ). Paired-samples t-tests with Bonferroni corrections revealed that serum B-ALP was significantly greater in WBV compared to WBV + BFR at POST $24 \mathrm{H}(p=0.028, d=0.31)$. The absolute mean change of serum B-ALP from pre-exercise values is presented in $\mathbf{F i g}$. $\mathbf{3 c}$.

- Table 2 Comparison of energy and nutrient intake data derived from food diaries completed over the 72 hours preceding both experimental trials $(n=10)$.

\begin{tabular}{|l|c|c|c|}
\hline & WBV & WBV+ BFR & \multirow{2}{*}{ p-value } \\
\cline { 2 - 3 } & Mean \pm SD & Mean \pm SD & \\
\hline Energy intake $(\mathrm{kJ})$ & $8121 \pm 1702$ & $8355 \pm 2204$ & 0.48 \\
\hline Carbohydrate $(\mathrm{g})$ & $221 \pm 39$ & $213 \pm 57$ & 0.53 \\
\hline Protein $(\mathrm{g})$ & $91 \pm 34$ & $92 \pm 29$ & 0.78 \\
\hline Calcium $(\mathrm{mg})$ & $966 \pm 243$ & $1096 \pm 396$ & 0.32 \\
\hline Vitamin D $(\mu \mathrm{g})$ & $1.9 \pm 1.3$ & $2.6 \pm 1.7$ & 0.14 \\
\hline $\begin{array}{l}\text { Note: WBV, whole-body vibration; WBV + BFR, whole-body vibration } \\
\text { with blood flow restriction. P-value indicates no between-trial } \\
\text { differences assessed using paired-samples t-test. }\end{array}$ \\
\hline
\end{tabular}


- Table 3 Descriptive data for all bone biomarker responses measured before and after whole-body vibration exercise with and without blood flow restriction $(n=10)$.

\begin{tabular}{|c|c|c|c|c|c|c|c|c|}
\hline & \multicolumn{4}{|c|}{ WBV } & \multicolumn{4}{|c|}{$W B V+B F R$} \\
\hline & PRE & POST & POST 1H & POST 24H & PRE & POST & POST 1H & POST 24H \\
\hline Sclerostin $\left(\mathrm{ng} \cdot \mathrm{mL}^{-1}\right)$ & $0.418 \pm 0.193$ & $0.460 \pm 0.240$ & $0.448 \pm 0.251$ & $0.433 \pm 0.199$ & $0.404 \pm 0.205$ & $0.425 \pm 0.192$ & $0.403 \pm 0.162$ & $0.439 \pm 0.186$ \\
\hline $95 \% \mathrm{Cl}\left(\mathrm{ng} \cdot \mathrm{mL}^{-1}\right)$ & $0.281-0.556$ & $0.289-0.632$ & $0.268-0.628$ & $0.291-0.575$ & $0.257-0.550$ & $0.288-0.562$ & $0.287-0.519$ & $0.304-0.574$ \\
\hline Change from PRE (\%) & - & $9.9 \pm 17.8$ & $7.1 \pm 19.6$ & $5.3 \pm 19.3$ & - & $9.8 \pm 22.2$ & $9.5 \pm 33.2$ & $17.7 \pm 32.0$ \\
\hline $\mathrm{CTX}\left(\mathrm{ng} \cdot \mathrm{mL}^{-1}\right)$ & $0.610 \pm 0.452$ & $0.598 \pm 0.415$ & $0.529 \pm 0.350$ & $0.648 \pm 0.450$ & $0.566 \pm 0.337$ & $0.535 \pm 0.307$ & $0.504 \pm 0.333$ & $0.514 \pm 0.299$ \\
\hline $95 \% \mathrm{Cl}\left(\mathrm{ng} \cdot \mathrm{mL}^{-1}\right)$ & $0.287-0.933$ & $0.301-0.895$ & $0.278-0.779$ & $0.326-0.969$ & $0.325-0.806$ & $0.316-0.755$ & $0.265-0.742$ & $0.300-0.728$ \\
\hline Change from PRE (\%) & - & $0.9 \pm 16.0$ & $-10.9 \pm 15.7$ & $6.1 \pm 20.6$ & - & $-3.3 \pm 18.2$ & $-11.7 \pm 22.5$ & $-7.7 \pm 24.5$ \\
\hline B-ALP $(\mu L)$ & $23.35 \pm 7.64$ & $24.18 \pm 7.71$ & $24.87 \pm 8.10$ & $25.94 \pm 8.82^{*}$ & $24.42 \pm 9.07$ & $25.35 \pm 8.14$ & $24.90 \pm 9.10$ & $23.47 \pm 8.29$ \\
\hline $95 \% \mathrm{Cl}(\mu \mathrm{L})$ & $17.89-28.82$ & $18.66-29.70$ & $19.08-30.66$ & $19.63-32.25$ & $17.93-30.90$ & $19.52-31.17$ & $18.39-31.42$ & $17.54-29.40$ \\
\hline Change from PRE (\%) & - & $4.1 \pm 6.0$ & $6.8 \pm 5.2$ & $11.0 \pm 6.8$ & - & $6.0 \pm 9.2$ & $2.5 \pm 8.7$ & $-3.4 \pm 2.4$ \\
\hline
\end{tabular}

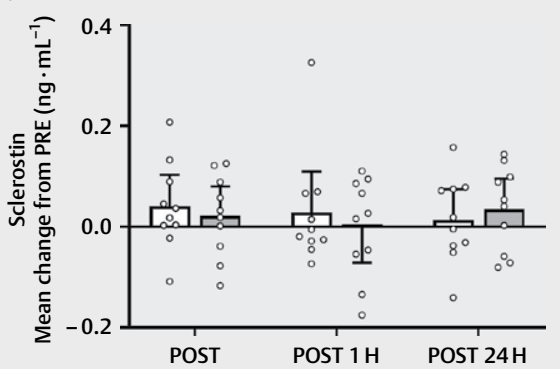

b

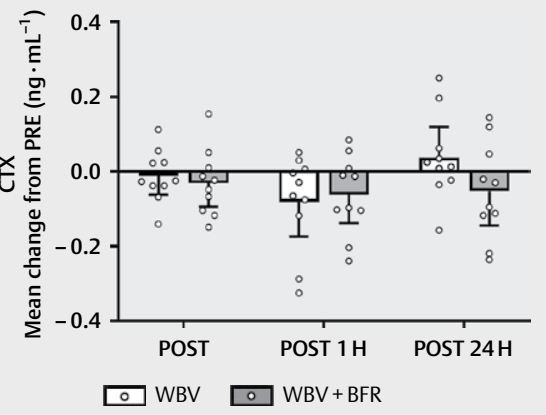

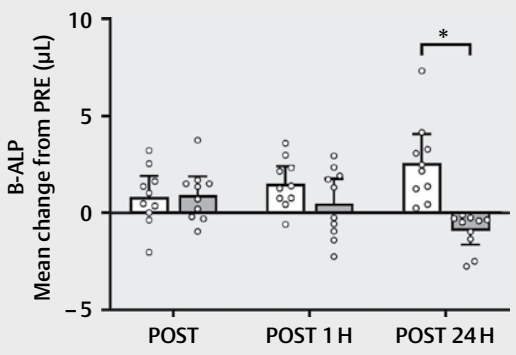

- Fig. 3 Mean absolute change of a serum sclerostin, $\mathbf{b}$ serum cross-linked C-terminal telopeptide of type 1 collagen (CTX), and $\mathbf{c}$ serum bonespecific alkaline phosphatase (B-ALP) from pre-exercise values following whole-body vibration exercise (WBV) and whole-body vibration exercise with blood flow restriction (WBV +BFR). Bars represent mean $\pm 95 \%$ confidence intervals, and open symbols are individual data $(n=10)$. POST, immediately post-exercise; POST 1H, 1-hour post-exercise; POST 24H, 24 hours post-exercise * Indicates a significant between-condition difference $(p<0.05)$.

\section{Discussion}

The primary aim of this study was to investigate the responses of sclerostin and bone turnover biomarkers to an acute bout of WBV superimposed with BFR. To achieve this aim, we controlled dietary intake and physical activity before test days and evaluated serum concentrations of sclerostin and biomarkers of bone turnover at periodic time points up to 24 hours after exercise. In contrast to our hypothesis, we found no significant changes in sclerostin or biomarkers of bone turnover in response to a single session of blood flow-restricted WBV exercise.

Few human studies have examined changes in sclerostin in response to exercise. A transient increase in serum sclerostin has been reported following an acute bout of WBV [22], combined resistance training with WBV [23], physical activity [18, 19], high-intensity interval exercise [20], and jumping [21]. One study found a lack of change in sclerostin immediately following five 1-minute bouts of WBV $(20 \mathrm{~Hz}, 3.38 \mathrm{~mm}, \sim 2.7 \mathrm{~g})$ [23], whereas another study reported a $91 \%$ increase in plasma sclerostin from pre- to 10 minutes post-WBV (40 Hz, $2 \mathrm{~mm}, \sim 2.7 \mathrm{~g})$ [22]. The contrast in findings may be attributed to the differences in the time point analyses or factors relating to the vibration stimulus. Bone cellular responses to mechanostimulation are influenced by the specific components of the imposed stressor [39]. Thus, between-study disparities in vibration variables such as frequency $(30 \mathrm{~Hz}$ versus $40 \mathrm{~Hz}$ for the present study and Cidem et al. [22], respectively) may explain the differential sclerostin response to WBV. It is worth noting that, based on the peak acceleration equation by Rauch et al. [29], the vibration stimulus applied in the study by Cidem et al. [22] may actually equate to an acceleration level of $6.4 \mathrm{~g}$, rather than the $2.7 \mathrm{~g}$ reported. This may suggest that the sclerostin response to WBV exercise is largely governed by the overall magnitude of the vibration stimulus. Further research is needed to determine the influence of the various vibration parameters (frequency, displacement, acceleration, and dose) on serum sclerostin over an extended time course.

Interestingly, we found that serum B-ALP values at 24 hours post-exercise were significantly greater following WBV compared to WBV + BFR. The acute rise in serum B-ALP 24 hours following 
WBV is suggestive of a favourable bone metabolic shift towards enhanced bone formation, particularly when considered alongside a non-significant change in CTX. To our knowledge, no other study has examined the response of B-ALP to WBV for up to 24 hours postexercise. When combined with resistance exercise, previous studies have shown that B-ALP either increases or remains unchanged $30 \mathrm{mi}$ nutes post-exercise $[40,41]$. It is possible that a longer time-window may be required to detect changes in serum B-ALP in response to WBV. In support of this, other studies have found significant increases in the bone formation marker osteocalcin following WBV, which peaked at 48 hours post-exercise [42].

No additive effect of BFR on the response of B-ALP or CTX to WBV was observed at any time point. These findings are likely due to components of the BFR stimulus (e.g. frequency, cuff size, fixed cuff pressure, restriction time, intermittent protocol) not being optimal to elicit bone cellular responses. One variable that may explain our lack of significant findings is the magnitude of BFR attained during exercise. Acute and chronic training studies that have shown BFR-induced changes in CTX and B-ALP have typically used elastic cuffs $5 \mathrm{~cm}$ in width with absolute cuff pressures in the range of $\sim 160-230 \mathrm{mmHg}$ [10-12]. In the present study, we used a cuff $13 \mathrm{~cm}$ wide and an absolute cuff pressure of $110 \mathrm{mmHg}$, as the cuff pressure required to occlude lower-body arterial blood flow has been reported to be approximately $40 \%$ lower when using wider cuffs compared to narrow cuffs [32]. Notably, another BFR study utilising a similar study population and cuff width $(13.5 \mathrm{~cm})$ reported that a cuff pressure of $139.75 \pm 14.41 \mathrm{mmHg}$ was enough to occlude lower-body blood flow [43]. Current literature suggests that the cuff pressure required to occlude blood flow to a limb (i. e. arterial occlusion pressure; AOP) is dependent on cuff properties (e. g. tourniquet shape, width, and length) and individual blood pressure and limb characteristics. Hence, it is suggested that cuff pressures should be set to a percentage of an individual's AOP, with cuff pressures equating to $50-80 \%$ of AOP typically recommended to support the efficacy of BFR training [2]. Based on previous observations, we estimate that our selected restriction pressure of $110 \mathrm{mmHg}$ restricted lower-limb blood flow by $\sim 60 \%$ [33]. This considered, it is possible that a cuff pressure of $110 \mathrm{mmHg}$ was not high enough to elicit any bone cellular responses. In addition, a fixed BFR pressure may result in different levels of BFR within the sample of the study, which may, consequently, reflect distinct exercise-induced physiological responses and therefore increase variability. Future studies should consider setting cuff pressure as a relative percentage of an individual's AOP to standardise restriction pressure across participants and mitigate inter-individual variability in BFR-induced physiological responses. Future research should also seek to clarify if different restriction pressures are requisite for stimulating bone cellular responses taking into consideration the discomfort and safety of the cuff pressure in the long-term.

Several physiological mechanisms by which BFR training may influence bone metabolism and evoke chronic skeletal adaptations have been proposed, including increased intramedullary pressure and interstitial fluid flow within the bone during BFR [44]. Additionally, sustained BFR-induced hypoxia may upregulate bone remodelling-related genes and induce downstream changes in bone cellular activity [45]. One factor that may influence the magnitude of localised hypoxia distal to the site of the cuff is the restriction time.
Previous studies that have reported BFR-induced changes in bone turnover biomarkers have used continuous $[10,11]$ and intermittent BFR protocols [12], and a minimum restriction time of 6-15 minutes per exercise. In contrast, the present study utilised an intermittent BFR protocol of ten cycles of 1 minute inflation/30 seconds deflation. Therefore, the chosen restriction time may not have been of sufficient duration to create any meaningful degree of localised hypoxia. A restriction time of a minimum of 5 minutes per exercise is recommended to promote improvements in muscle strength and hypertrophy [2], although no such guidelines for skeletal adaptations currently exist. Based on the conflicting findings between our study and others, it is possible that longer restriction times may be required to promote bone cellular responses. Prolonged restriction times would likely extend the duration of the acute state of hypoxia in the tissues below the cuff, possibly causing alterations of bone bioenergetics and upregulation of bone remodelling-related genes through the stabilisation of hypoxic-induced gene transcription factors (HIFs) and activation of the HIF pathway [46].

The decision to apply an intermittent BFR protocol with short restriction times was informed by prior findings that a prolonged mechanical stimulus leads to an exponential reduction in bone mechanosensitivity that diminishes the osteogenic response to loading [36]. Intermittent mechanical loading interspersed with rest periods has been reported to elicit greater bone responses than continuous mechanical loading in animal models [47-49], potentially due to the inclusion of brief rest intervals that allow the bone to "resensitise" to the loading stimulus [50]. Our rationale for deflating the cuffs during the rest period was an attempt to mimic cyclic loading that has been reported to influence fluid flow and intramedullary pressure sensed by osteocytes that release sclerostin [51]. However, this may have been a limiting factor in being able to detect a difference between the two protocols. Previous research has shown the addition of BFR to an acute bout of WBV increases myoelectric activity [52,53], metabolite accumulation [52, 53], and serum levels of growth hormone [53] to a greater extent than WBV exercise alone when the cuff pressure was inflated during rest periods. Since muscle activity and hypoxia-driven physiological changes may influence bone metabolism [54, 55], future studies may consider keeping the cuff pressure inflated during the entire exercise including rest periods, yet also consider the discomfort of the cuff pressure.

This study is novel in that it is the first study to investigate the acute response of serum sclerostin and biomarkers of bone turnover to WBV with BFR. The strengths of this study include the crossover design with random assignment of WBV or combined WBV and BFR. To account for the circadian variation in bone biomarkers $[56,57]$, the exercise protocol and venous blood sampling were performed at the same time of day for both experimental conditions, and we measured the bone biomarker response up to 24 hours after exercise. However, this study also has several limitations that must be considered when interpreting the findings. Firstly, our study population consisted of untrained but otherwise healthy adult males so the present findings should not be extrapolated to other study populations, particularly those subject to skeletal weakness, such as postmenopausal women and older adults. Secondly, the semi-squat position was standardised by a goniome- 
tric assessment of knee joint angle, but other biomechanical parameters such as joint angle at the hip and ankle may influence the transmission of the vibration stimulus. Thirdly, we did not include a BFR-only control condition that would have provided insight into the overall contribution of the BFR stimulus to the reported bone biomarker responses. Fourthly, we used B-ALP and CTX as proxy measures of bone formation and resorption, respectively, in line with similar studies that have investigated the acute bone turnover response to exercise $[11,40,41]$. Future clinical investigations into the response of bone turnover to BFR strategies should consider using recommended biomarkers of bone formation, such as $\mathrm{N}$ terminal propeptide of type 1 procollagen (P1NP) [58]. Finally, while bone biomarkers are commonly used to provide a "snapshot" of the bone turnover response to exercise, they are affected by various endogenous and exogenous sources of variability, including pre-exercise feeding and loading history, that may mask the true dynamics of bone metabolism [59]. To mitigate this, we asked participants to replicate their diet and activity during the 72 hours leading up to the experimental trials, but more rigorous standardisations could be considered in future studies.

In conclusion, the present findings suggest that the addition of BFR to ten 1-minute bouts of WBV elicited no significant change in the serum concentration of sclerostin, CTX, or B-ALP at any timepoint up to 24 hours post-exercise in untrained, adult men. Future investigations into the effects of combined WBV and BFR exercise on bone metabolism should consider the parameters and overall intensity of the vibration stimulus, the methodological factors that influence BFR such as standardisation of restriction pressures while considering the discomfort and safety of the cuff pressure, and include populations at risk of skeletal weakness such as postmenopausal women. Further efforts should be made to fully elucidate the osteogenic potential of BFR, and whether it may be beneficial to perform in conjunction with other exercise modalities.

\section{Funding}

This study was funded by the University of Surrey Pump Priming Award.

\section{Acknowledgements}

The authors would like to sincerely thank Miss Lindsay Bamford and Dr. Fariba Shoejaee-Moradie in the School of Biosciences at the University of Surrey for their support with the biochemical assays, and the participants for taking part in the study.

\section{Conflict of Interest}

The authors declare that they have no conflict of interest.
References

[1] Scott BR, Loenneke JP, Slattery KM et al. Blood flow restricted exercise for athletes: A review of available evidence. J Sci Med Sport 2016; 19: 360-367

[2] Scott BR, Loenneke JP, Slattery KM et al. Exercise with blood flow restriction: An updated evidence-based approach for enhanced muscular development. Sports Med 2015; 45: 313-325

[3] Slysz J, Stultz J, Burr JF. The efficacy of blood flow restricted exercise: A systematic review \& meta-analysis. J Sci Med Sport 2016; 19: 669-675

[4] Hughes L, Paton B, Rosenblatt B et al. Blood flow restriction training in clinical musculoskeletal rehabilitation: A systematic review and meta-analysis. Br J Sports Med 2017; 51: 1003-1011

[5] Delmas PD, Eastell R, Garnero P et al. The use of biochemical markers of bone turnover in osteoporosis. Osteoporos Int 2000; 11: S2-S17

[6] Okuno S, Inaba M, Kitatani K et al. Serum levels of C-terminal telopeptide of type I collagen: a useful new marker of cortical bone loss in hemodialysis patients. Osteoporos Int 2005; 16: 501-509

[7] Ueda M, Inaba M, Okuno $S$ et al. Serum BAP as the clinically useful marker for predicting BMD reduction in diabetic hemodialysis patients with low PTH. Life Sci 2005; 77: 1130-1139

[8] Johansson $\mathrm{H}$, Odén A, Kanis JA et al. A meta-analysis of reference markers of bone turnover for prediction of fracture. Calcif Tissue Int 2014; 94: 560-567

[9] Banfi G, Lombardi G, Colombini A et al. Bone metabolism markers in Sport Med. Sports Med 2010; 40: 697-714

[10] Beekley MD, Sato Y, Abe T. KAATSU-walk training increases serum bone-specific alkaline phosphatase in young men. Int J KAATSU Training Res 2005; 1: 77-81

[11] Bemben DA, Palmer IJ, Abe T et al. Effects of a single bout of low intensity KAATSU resistance training on markers of bone turnover in young men. Int J KAATSU Training Res 2007; 3: 21-26

[12] Karabulut M, Bemben DA, Sherk VD et al. Effects of high-intensity resistance training and low-intensity resistance training with vascular restriction on bone markers in older men. Eur J Appl Physiol 2011; 111: 1659-1667

[13] Robling AG, Niziolek PJ, Baldridge LA et al. Mechanical stimulation of bone in vivo reduces osteocyte expression of Sost/sclerostin. J Biol Chem 2008; 283: 5866-5875

[14] Bloomfield SA. Does altered blood flow to bone in microgravity impact on mechanotransduction? J Musculoskelet Neuronal Interact 2006; 6: 324-326

[15] Fritton SP, Weinbaum S. Fluid and solute transport in bone: flow-induced mechanotransduction. Annu Rev Fluid Mech 2009; 41: 347-374

[16] Lin C, Jiang X, Dai Z et al. Sclerostin mediates bone response to mechanical unloading through antagonizing $\mathrm{Wnt} / \beta$-catenin signaling. J Bone Miner Res 2009; 24: 1651-1661

[17] Poole KES, Van Bezooijen RL, Loveridge $N$ et al. Sclerostin is a delayed secreted product of osteocytes that inhibits bone formation. FASEB J 2005; 19: 1842-1844

[18] Pickering ME, Simon M, Sornay-Rendu E et al. Serum sclerostin increases after acute physical activity. FASEB J 2017; 101: 170-173

[19] Gombos GC, Bajsz V, Pék E et al. Direct effects of physical training on markers of bone metabolism and serum sclerostin concentrations in older adults with low bone mass. BMC Musculoskelet Disord 2016; 17: 254

[20] Kouvelioti R, Kurgan N, Falk B et al. Response of sclerostin and bone turnover markers to high intensity interval exercise in young women: Does impact matter? Biomed Res Int 2018; 4864952

[21] Falk B, Haddad F, Klentrou P et al. Differential sclerostin and parathyroid hormone response to exercise in boys and men. Osteoporos Int 2016; $27:$ 1245-1249 
[22] Çidem M, Karakoç Y, Ekmekçi $\mathrm{H}$ et al. Effects of whole-body vibration on plasma sclerostin level in healthy women. Turk J Med Sci 2014; 44: 404-410

[23] Sharma-Ghimire P, Chen Z, Sherk V et al. Sclerostin and parathyroid hormone responses to acute whole-body vibration and resistance exercise in young women. J Bone Miner Metab 2019; 37: 358-367

[24] Rubin C, Turner AS, Mallinckrodt C et al. Mechanical strain, induced noninvasively in the high-frequency domain, is anabolic to cancellous bone, but not cortical bone. Bone 2002; 30: 445-452

[25] Kelly PJ, Bronk JT. Venous pressure and bone formation. Microvasc Res 1990; 39: 364-375

[26] Weeks BK, Beck BR. The BPAQ: A bone-specific physical activity assessment instrument. Osteoporos Int 2008; 19: 1567-1577

[27] Harriss DJ, Macsween A, Atkinson G. Ethical standards in sport and exercise science research: 2020 update. Int J Sports Med 2019; 40: 813-817

[28] Oliveira LC, Oliveira RG, Pires-Oliveira DAA. Effects of whole body vibration on bone mineral density in postmenopausal women: A systematic review and meta-analysis. Osteoporos Int 2016; 27 : 2913-2933

[29] Rauch F, Sievanen H, Boonen S et al. Reporting whole-body vibration intervention studies: Recommendations of the International Society of Musculoskeletal and Neuronal Interactions. J Musculoskelet Neuronal Interact 2010; 10: 193-198

[30] Centner C, Ritzmann R, Gollhofer A et al. Effects of whole-body vibration training and blood flow restriction on muscle adaptations in women: a randomized controlled trial. J Strength Cond Res 2020; 34: 603-608

[31] Aguayo D, Mueller SM, Boutellier U et al. One bout of vibration exercise with vascular occlusion activates satellite cells. Exp Physiol 2016; 101: 295-307

[32] Loenneke JP, Fahs CA, Rossow LM et al. Effects of cuff width on arterial occlusion: implications for blood flow restricted exercise. Eur J Appl Physiol 2012; 112: 2903-2912

[33] Hunt JEA, Stodart C, Ferguson RA. The influence of participant characteristics on the relationship between cuff pressure and level of blood flow restriction. Eur J Appl Physiol 2016; 116: 1421-1432

[34] Hewitt JD, Harrelson JM, Dailiana Z et al. The effect of intermittent pneumatic compression on fracture healing. J Orthop Trauma 2005; 19: 371-376

[35] Park SH, Silva M. Effect of intermittent pneumatic soft-tissue compression on fracture-healing in an animal model. J Bone Joint Surg Am 2003; 85: 1446-1453

[36] Turner CH, Robling AG. Exercises for improving bone strength. $\mathrm{Br}$ ] Sports Med 2005; 39: 188-189

[37] Cohen J. Statistical Power Analysis for the Behavioral Sciences. 2nd ed. Hillsdale: Lawrence Erlbaum; 1988

[38] Lakens D. Calculating and reporting effect sizes to facilitate cumulative science: A practical primer for t-tests and ANOVAs. Front Psychol 2013; 4: 863

[39] Rittweger ]. Vibration as an exercise modality: how it may work, and what its potential might be. Eur J Appl Physiol 2010; 108: 877-904

[40] Bemben DA, Sharma-Ghimire P, Chen Z et al. Effects of whole-body vibration on acute bone turnover marker responses to resistance exercise in young men. J Musculoskelet Neuronal Interact 2015; 15: 23-31
[41] Sherk VD, Chrisman C, Smith J et al. Acute bone marker responses to whole-body vibration and resistance exercise in young women. J Clin Densitom 2013; 16: 104-109

[42] Bowtell JL, Jackman SR, Scott S et al. Short duration small sided football and to a lesser extent whole body vibration exercise induce acute changes in markers of bone turnover. Biomed Res Int 2016; 2016: 3574258

[43] Freitas EDS, Miller RM, Heishman AD et al. Acute physiological responses to resistance exercise with continuous versus intermittent blood flow restriction: a randomized controlled trial. Front Physiol 2020; 11: 132

[44] Loenneke JP, Young KC, Fahs CA et al. Blood flow restriction: Rationale for improving bone. Med Hypotheses 2012; 78: 523-527

[45] Wan C, Shao J, Gilbert SR et al. Role of HIF-1 $\alpha$ in skeletal development. Ann N Y Acad Sci 2010; 1192: 322-326

[46] Camacho-Cardenosa M, Camacho-Cardenosa A, Timón R et al. Can hypoxic conditioning improve bone metabolism? A systematic review. Int J Environ Res Public Health 2019; 16: 1799

[47] Robling AG, Burr DB, Turner CH. Recovery periods restore mechanosensitivity to dynamically loaded bone. J Exp Biol 2001; 204: 3389-3399

[48] Srinivasan S, Agans SC, King KA et al. Enabling bone formation in the aged skeleton via rest-inserted mechanical loading. Bone 2003; 33: 946-955

[49] Srinivasan S, Ausk BJ, Poliachik SL et al. Rest-inserted loading rapidly amplifies the response of bone to small increases in strain and load cycles. J Appl Physiol (1985) 2007; 102: 1945-1952

[50] Srinivasan S, Ausk B], Bain SD et al. Rest intervals reduce the number of loading bouts required to enhance bone formation. Med Sci Sports Exerc 2015; 47: 1095-1103

[51] Gross TS, Poliachik SL, Ausk B] et al. Why rest stimulates bone formation: a hypothesis based on complex adaptive phenomenon. Exerc Sport Sci Rev 2004; 32: 9-13

[52] Centner C, Ritzmann R, Schur S et al. Blood flow restriction increases myoelectric activity and metabolic accumulation during whole-body vibration. Eur J Appl Physiol 2019; 119: 1439-1449

[53] Cai ZY, Chen WC, Wu CM. Acute effects of whole body vibration combined with blood restriction on electromyography amplitude and hormonal responses. Biol Sport 2018; 35: 301-307

[54] Herrmann M, Engelke K, Ebert R et al. Interactions between muscle and bone-where physics meets biology. Biomolecules 2020; 10: 432

[55] Rankin EB, Giaccia AJ, Schipani E. A central role for hypoxic signaling in cartilage, bone, and hematopoiesis. Curr Osteoporos Rep 2011; 9: 46-52

[56] Redmond J, Fulford A], Jarjou L et al. Diurnal rhythms of bone turnover markers in three ethnic groups J Clin Endocrinol Metab 2016; 101: 3222-3230

[57] Swanson C, Shea SA, Wolfe P et al. 24-hour profile of serum sclerostin and its association with bone biomarkers in men. Osteoporos Int 2017; 28: 3205-3213

[58] Eastell R, Pigott T, Gossiel F et al. Diagnosis of endocrine disease: bone turnover markers: are they clinically useful? Eur ] Endocrinol 2018; 178: R19-R31

[59] Hlaing TT, Compston JE. Biochemical markers of bone turnover - uses and limitations. Ann Clin Biochem 2014; 51: 189-202 